\title{
Acid Hydrolysis of Proteins in Matrix Assisted Laser Desorption Ionization Matrices
}

\author{
Elizabeth Remily-Wood, ${ }^{\text {a Hayley Dirscherl, }}{ }^{\text {a,b }}$ and John M. Koomen ${ }^{\mathrm{a}, \mathrm{c}}$ \\ ${ }^{a}$ Molecular Oncology and Proteomics, H. Lee Moffitt Cancer Center and Research Institute, Tampa, Florida, \\ USA \\ ${ }^{\mathrm{b}}$ Biomedical Engineering, Columbia University, New York, USA \\ ${ }^{c}$ Departments of Chemistry, Molecular Medicine, and Oncologic Sciences University of South Florida, \\ Tampa, Florida, USA
}

Sample preparation is crucial to the success of experiments in biological mass spectrometry. In proteomics, digestion of the proteins into peptides is a key step for "bottom-up" approaches. Often, the use of enzymes requires physiological conditions, producing peptides that must be extracted or further purified before mass analysis. Chemical cleavage reagents offer more flexibility and can be more compatible with downstream mass analysis. Expanding on prior work using acid hydrolysis, proteolysis with matrix-assisted laser desorption ionization (MALDI) matrices is presented. This sample preparation can be performed rapidly with a minimum of reagents and sample handling, but it must first be evaluated in terms of digestion efficiency, missed cleavages, and side reactions before implementation for in-gel digestion and in-solution digestion using minimal volumes of protein. Time courses of acid hydrolysis are shown for protein standards, illustrating the sensitivity of this type of sample preparation, minimization of side reactions, and performance for proteins in mixtures. To illustrate the potential for sensitive detection of a specific protein, MALDI matrix hydrolysis is used to digest a protein immunoprecipitated from cell lysate. (J Am Soc Mass Spectrom 2009, 20, 2106-2115) (c) 2009 Published by Elsevier Inc. on behalf of American Society for Mass Spectrometry

$\mathrm{P}$ roteolysis is often the critical step in preparing proteins for analysis with mass spectrometry. Because trypsin digestion is robust, predictable, inexpensive, and produces peptides amenable to mass spectrometry (both in basicity and molecular weight), it is the preferred reagent in most digestion protocols. Typical digestions proceed in solution or in gel for 16 to $24 \mathrm{~h}$ at $37^{\circ} \mathrm{C}$. This step is often arranged overnight, making it convenient for sample processing. Methods for improving or hastening trypsin digestion have been investigated digestion in mixed aqueous/organic solvent systems [1] and microwave heating, both in solution [2] and in gel [3]. The combination of mixed solvent systems and microwave assisted digestion has also been reported to improve enzymatic proteolysis [4]. Recent advances have combined prior techniques with immobilized trypsin microspheres [5]. In addition to improving trypsin digestion, the use of other enzymes or chemical cleavage agents is necessary to produce complementary or, in some cases, more comprehensive sequence information.

Acid hydrolysis is one example of a complementary digestion technique. While strong acids have been used to degrade proteins for amino acid analysis [6], weak

Address reprint requests to Dr. J. M. Koomen, Molecular Oncology/ Proteomics SRB3, H. Lee Moffitt Cancer Center and Research Institute, 12902 Magnolia Drive, Tampa, FL 33612, USA. E-mail: john.koomen@ moffitt.org acids (e.g., formic and acetic) can be used to cleave proteins specifically at aspartic acid residues (Asp). Building on prior work in proteolytic digestion using acid hydrolysis [7], the coupling of Asp-directed proteolysis and mass spectrometry was reported by Li et al. using $2 \%$ formic acid at elevated temperatures [8]. Similar to improvements in trypsin digestion, microwave-assisted acid hydrolysis (MAAH) has been shown to speed the formation of peptide fragments when compared with the original in solution method [9]. MAAH methods have also been demonstrated for $25 \%$ trifluoroacetic acid [10] and $12.5 \%$ acetic acid [11, 12]. These methods are compatible with downstream liquid chromatography and mass spectrometry, and they have been applied to the identification of bacterial spores [13] and viruses [14], as well as proteome analysis of yeast ribosomes [11, 12] and zebrafish liver [15].

While, previous work on acid hydrolysis has focused on optimization and adaptation to proteome-wide applications, the use of acid hydrolysis in targeted mass spectrometry experiments for detection of specific proteins could also prove to be valuable. While trypsin digestions produce peptides with C-terminal lysine and arginine, few internal basic residues, peptides created by acid hydrolysis can contain clusters of basic residues, which may provide an advantage in detecting lower abundance proteins in mixtures. The strong MALDI ion signal from a peptide with multiple basic residues may 
be detectable even against a background of peptides at higher concentration, due to signal enhancement from higher proton affinity.

To develop an acid hydrolysis method for targeted peptide detection and quantification, the reproducibility and effectiveness of the digest must be evaluated. Furthermore, the reaction volumes must be minimized, to maximize the concentration of the protein(s) of interest. Ideally, small volumes of concentrated proteins could be proteolyzed and spotted directly on the MALDI target, effectively processing and mass analyzing the entire sample. To that end, acid hydrolysis in matrix-assisted laser desorption ionization (MALDI) matrix solutions is reported and compared with other acid hydrolysis reagents and trypsin. These methods enable protein detection with a minimum of reagents and sample handling, eliminating the sample loss that can occur due to microcentrifuge tube to vial transfers or dilution and concentration that are frequently included in tryptic digestion protocols. Peptides and proteins are digested with MALDI matrices under different conditions to evaluate the feasibility and potential utility of this technique.

\section{Materials and Methods}

All protein standards and chemicals were purchased from Sigma (St. Louis, MO, USA) or Aldrich (Milwaukee, WI, USA), unless otherwise noted. Acetonitrile and HPLC grade water were purchased from Burdick and Jackson (Honeywell, Muskegon, MI, USA). The acids used in this study are listed in Table 1 with their $\mathrm{pKa}$ values [16, 17] and concentrations in the reaction solutions.

All reactions were carried out at $99^{\circ} \mathrm{C}$ using a PCR machine (Mastercycler Eppendorf, Westbury, NY, USA) in $0.5 \mathrm{~mL}$ tubes with $100 \mu \mathrm{L}$ reaction volumes unless otherwise noted. Peptides and proteins were hydrolyzed in aqueous solutions of acetic, formic, or trifluoroacetic acid in $10 \%, 5 \%, 2 \%, 1 \%$, and $0.1 \%$ volumeto-volume ratios. Common MALDI matrices for peptides were also tested for acid hydrolysis at

Table 1. Reagents used for acid hydrolysis of proteins, listed by the negative $\log$ of their aqueous dissociation constants. MALDI matrices are listed in italics. The superscripts indicate the references for the $\mathrm{pKa}$ values

\begin{tabular}{lcr}
\hline \multicolumn{1}{c}{ Acid name (abbreviation) } & $\begin{array}{c}\text { pKa } \\
\text { (aqueous) }\end{array}$ & $\begin{array}{c}\text { Concentration } \\
(\mathrm{mM})\end{array}$ \\
\hline \hline Trifluoroacetic acid (TFA) & $0.50^{16}$ & $13-260$ \\
$\alpha$-Cyano-4-hydroxycinnamic acid & $1.17^{16}$ & $5-105$ \\
$\quad$ (CHCA) & & \\
2,5-Dihydroxybenzoic acid (DHB) & $3.01^{16}$ & $160-320$ \\
$\begin{array}{l}\text { Formic acid (FA) } \\
\text { Sinapinic acid 3,5-dimethoxy-4- }\end{array}$ & $3.75^{17}$ & $26-530$ \\
$\quad$ hydroxycinnamic acid & $3.98^{16}$ & $110-220$ \\
$\begin{array}{l}\text { Ferulic acid trans-4-hydroxy-3- } \\
\quad \text { methoxycinnamic acid }\end{array}$ & $4.04^{16}$ & $130-260$ \\
Acetic acid (AA) & $4.76^{17}$ & $17-330$ \\
\hline
\end{tabular}

varying concentrations, typically ranging from 1 to 20 $\mathrm{mg} / \mathrm{mL}$, including $\alpha$-cyano-4-hydroxycinnamic acid (CHCA), 2,5-dihydroxybenzoic acid (DHB) in either $50 \%$ aqueous ACN or $100 \%$ water, 3,5-dimethoxy-4hydroxycinnamic acid (sinapinic acid), and trans-4hydroxy-3-methoxycinnamic acid (ferulic acid). Most acid hydrolysis reactions were performed using 5 to 10 $\mathrm{mg} / \mathrm{mL}$ matrix solutions in $50 \%$ acetonitrile. Methanol cannot be used because the methylation of the acidic side chains proceeds at similar rates to acid hydrolysis, leading to $\sim 50 \%$ cleavage and $50 \%$ methyl ester formation. Low volume reactions $(5 \mu \mathrm{L})$ were performed using modifiers similar to those described by Palmblad and Cramer [18]. An acid hydrolysis solvent system containing $5 \mathrm{mg} / \mathrm{mL}$ CHCA and $10 \mu \mathrm{L} / \mathrm{mL}$ glycerol in a $50 \%$ aqueous $\mathrm{ACN}$ was found to prevent evaporation and facilitate acid hydrolysis without adding significant chemical background noise to the MALDI mass spectra. In each incubation, aliquots were extracted for MALDI mass analysis at selected time points.

Peptides, including human angiotensin I (DRVYIHPFHL), oxidized human insulin B chain, and synthetic epidermal growth factor receptor (EGFR) phosphopeptides, were hydrolyzed at $1 \mathrm{pmol} / \mu \mathrm{L}$ concentrations. Protein standards at $1 \mathrm{pmol} / \mu \mathrm{L}$ (bovine transferrin and chicken ovalbumin) were also cleaved over time (0 to $24 \mathrm{~h}$ ) to optimize acid hydrolysis in MALDI matrices. To further evaluate sensitivity, efficiency, and side reactions, bovine ubiquitin and transferrin were diluted in matrix solutions, to concentrations ranging from 500 $\mathrm{fmol} / \mu \mathrm{L}$ to $1 \mathrm{fmol} / \mu \mathrm{L}$ and hydrolyzed for timescales up to $1 \mathrm{~h}$ for ubiquitin and $2.5 \mathrm{~h}$ for transferrin. Bovine transferrin and chicken ovalbumin were also cleaved in $\mathrm{CHCA}, 2 \%$ formic acid (both in aqueous and 50\% ACN solutions) for $2 \mathrm{~h}$, these digests were then compared with tryptic digestions (both 2 and $18 \mathrm{~h}$ ) and overnight digestion with V-8 protease. All digests were then spotted in quintuplicate on a MALDI plate in amounts ranging from $250 \mathrm{fmol}$ to $1 \mathrm{fmol}$ per matrix deposit. The resulting peptide detection and sequence coverage were then compared between methods.

Mixtures of bovine ubiquitin and transferrin in 1:101:100 ratios were also hydrolyzed to evaluate the detection of the lower abundance component (ubiquitin). Duplicate reactions were done with the same protein ratios using trypsin as the enzyme. Peptides were extracted from an equimolar amount of reaction mixture using pipette tips packed with $\mathrm{C} 18$ reverse phase resin (Ziptip; Millipore, Billerica, MA, USA) after digestion and eluted directly onto a MALDI plate with 5 $\mathrm{mg} / \mathrm{mL}$ CHCA for sensitivity comparison.

For immunoprecipitation of $\beta$-catenin (CTNB1) from HCT115 cell lysates prepared from $10^{7}$ cells, the protein was pulled down using $50 \mu \mathrm{L}$ of protein A beads loaded with mouse anti- $\beta$-catenin antibody. After denaturation by boiling in Laemmli loading buffer with $5 \mathrm{mM}$ DTT for $10 \mathrm{~min}$, the proteins were separated by SDS PAGE and visualized with Coomassie blue G. Resulting bands were excised and destained in 50\% methanol in $50 \mathrm{mM}$ 
ammonium bicarbonate. In parallel with sodium dodecyl sulfate-polyacrylamide gel electrophoresis (SDSPAGE) separated protein standards, $\beta$-catenin bands were incubated in $50 \mu \mathrm{L}$ of $5 \mathrm{mg} / \mathrm{mL}$ CHCA for $3 \mathrm{~h}$ [7]. Resulting digests were then directly spotted and analyzed with MS.

Samples were analyzed in positive ion linear mode on a MALDI TOF mass spectrometer (Voyager; ABI, Framingham, MA, USA) with the following settings: 25 $\mathrm{kV}$ accelerating voltage, $94.5 \%$ grid voltage, and $350 \mathrm{~ns}$ delay. In each spectrum, 150 laser shots were accumulated and internally calibrated; data are presented for the average of at least three replicates. High-resolution MALDI mass spectra and tandem mass spectra were acquired on tandem-TOF mass spectrometer (4700; Applied Biosystems, Framingham, MA, USA). Each acquisition stored the accumulation of 1500 shots; samples were externally calibrated using a standard peptide mix provided by the manufacturer.

Data analysis was performed using measurements of signal-to-noise ratios or peak height generated by the software programs used for instrument control (Data Explorer and 4000 Series Explorer; Applied Biosystems, Framingham, MA, USA). Peak lists were exported for matching to theoretical peptides using the MASCOT algorithm (www.matrixscience.com) [19]. Searches were conducted with no enzyme specificity initially; then the enzymatic specificity was set strictly to those cleavages that were observed with modifications for C-terminal water loss and aspartic acid residue cleavage. Mascot parameters were set with the following: peptide tolerance: $30 \mathrm{ppm}$, up to 4 missed cleavages, and all data were searched against the SwissProt database. The Mascot score and sequence coverage are used to compare different digestion methods and optimize reaction times for each protein. Searches were performed using cleavage C-terminal to Asp and enabling C-terminal loss of aspartic acid and loss of water from selected residues. To detect ubiquitin in the mixtures with transferrin, predetermined peptide masses that had been verified with MS/MS were examined manually for ion signal.

\section{Results}

\section{MALDI Matrix Acid Hydrolysis of Peptides}

To evaluate the potential of acid hydrolysis with MALDI matrices, hydrolysis of the Angiotensin I peptide, DRVYIHPFHL, was performed with each acid and MALDI matrix; all data were compared against the established method using $2 \%$ formic acid [8]. This peptide model was chosen because only one specific cleavage site is present $\mathrm{C}$-terminal to the $\mathrm{N}$-terminal aspartic acid residue. Furthermore, the peptide has four basic amino acids, so the cleavage of the aspartic acid will not significantly change its detection in positive ion mode MALDI analysis. However, acid hydrolysis of angiotensin I may not go to completion because of interactions between aspartic acid residue and its neighboring arginine, similar to the observation that neighboring acidic residues produce missed cleavages in tryptic digests by forming salt bridges with lysine and arginine residues.

Each acid hydrolysis reaction was sampled at multiple time points over the first $4 \mathrm{~h}$. By measuring the amount of ion signals corresponding to DRVYIHPFHL at $m / z 1296.7$ and RVYIHPFHL at $m / z$ 1181.7, the reaction progress could be monitored. Salt adducts or side reaction products, such as water loss, could also be detected, but those ion signals were lower than $10 \%$ of the desired product. Lower mass peptide signals were also sequenced to examine nonspecific cleavage. By examining the total amount of peptide ion signal over time, the non-specific degradation of the peptide could also be assessed. The relative reaction rates calculated over the first $4 \mathrm{~h}$ of acid hydrolysis ranked as follows: formic acid $>$ trifluoroacetic acid $>\alpha$-cyano-4hydroxycinnamic acid $>$ acetic acid $>2,5$-dihydroxybenzoic acid $>$ sinapinic acid. No acid hydrolysis of angiotensin I was detected in solutions of ferulic acid. TFA was ruled out from future studies because non-specific cleavage products were observed in these reactions even at short time points. Acetic acid and the MALDI matrices produced specific cleavage $C$-terminal to the aspartic acid residue, similar to formic acid. While formylation becomes a significant side reaction in $2 \%$ formic acid hydrolysis, no similar side reactions were observed for acetic acid or any of the MALDI matrices. Although no trend can be reported between the $\mathrm{pKa}$ and reaction rate for the entire group of acids, the effectiveness of the MALDI matrices did appear to improve with decreasing $\mathrm{pKa}$. In the matrix digests, the resulting peptide ion signals were best in $\mathrm{CHCA}$; DHB and sinapinic acids produced the cleavage product, RVYIHPFHL, but the mass spectra were poorer in quality. These results may indicate that matrix crystallization and desorption/ionization are not effected by high incubation temperature $\left(\sim 100^{\circ} \mathrm{C}\right)$ for these acid hydrolysis reactions.

Optimization of digestion with CHCA matrix was pursued because the homogeneity of the dried droplet deposits and resulting ion signal quality are significantly better than DHB or sinapinic acid. The cleavage of angiotensin, DRVYIHPFHL, was compared in digests using $2 \%$ formic acid, $10 \mathrm{mg} / \mathrm{mL} \mathrm{CHCA}$, and $2 \%$ acetic acid. The overall cleavage is better at longer incubation times for formic acid hydrolysis than in CHCA solutions; however, long incubation times increase the amount of side reactions, including water loss and formylation of amines ( $\mathrm{N}$-termini and lysine residues). At incubation times less than $5 \mathrm{~h}$, no significant differences in product formation were observed between $2 \%$ formic acid and $10 \mathrm{mg} / \mathrm{mL} \mathrm{CHCA.} \mathrm{Al-}$ though the variability was higher in matrix digests, the average product formation was similar: $\sim 30 \%$ to $35 \%$ over the first $4 \mathrm{~h}$. Additional peptide digests with oxidized insulin $b$ chain indicate that acid hydrolysis 
can cleave C-terminal to cysteic acid residues (data not shown) and that phosphopeptides, like YSSDPTGALTEDSIDDTFLPVPEPYINQSVPK from human epidermal growth factor receptor, lose phosphorylation during proteolysis at levels of $50 \%$ or more during the timescale of the cleavage reaction. MALDI matrix acid hydrolysis of bovine insulin showed no effect on the disulfide bonds. Low levels of cleavage at glycine were observed, and they increased over time. This same pattern of glycine cleavage was observed in other standard proteins (OVAL_CHICK, TRFE_BOVIN, MYG_ HOUSE, and UBIQ_BOVIN), with, on average, cleavage occurring at $10 \%$ of possible glycine residues after $1 \mathrm{~h}$ of incubation. Other nonaspartic acid cleavages, such as cysteine, threonine, and serine were observed, however their occurrence was low (on average $0 \%$ to $5 \%$ under $2 \mathrm{~h}$ ). In total, cleavages at residues other than aspartic acid were less than $10 \%$ by frequency at these timescales. Additional peptide modifications including were included in the database searches; there was no evidence of pyroglutamic acid formation for the proteins hydrolyzed with MALDI matrix. A low occurrence of deamidation reactions was observed, which was similar to tryptic digestions.

\section{MALDI Matrix Acid Hydrolysis of Proteins}

To examine the performance of MALDI matrix in protein digestion, rapid acid hydrolysis with $10 \mathrm{mg} / \mathrm{mL}$ CHCA was performed on two standard proteins: chicken egg ovalbumin and bovine transferrin. Representative MALDI mass spectra are shown in Figure 1. After $30 \mathrm{~min}$, the ovalbumin digest showed several peaks that could be matched by $\mathrm{m} / \mathrm{z}$ to ovalbumin; peptide assignments were verified by MALDI tandem mass spectrometry (Mascot Score 180 with five peptides matched to MS/MS data). Eleven ovalbumin peaks were manually identified in Figure 1a at the following average $m / z$ values: $715.8,1182.2,1448.6,1564.7,1904.1$, 2164.5, 2279.6, 2579.1, 2597.1, 3882.5, and 4554.9. Transferrin digests also produced representative peptides after short incubations in CHCA. After a $2.5 \mathrm{~h}$ digestion, 16 transferrin peaks were assigned in Figure $1 \mathrm{~b}$ at the following average $\mathrm{m} / \mathrm{z}$ values: 1202.3, 1418.6, 1618.8, 1733.9, 1758.9, 1950.2, 2035.3, 2290.5, 2375.7, 2630.9, 2755.1, 3334.7, 3864.4, 4470.1, 5185.8, and 6325.2 (Mascot Score 235 with 10 peptides identified with MS/MS data). In addition, LC-MS/MS data were used to corroborate the cleavages and modifications; these are not

(a)

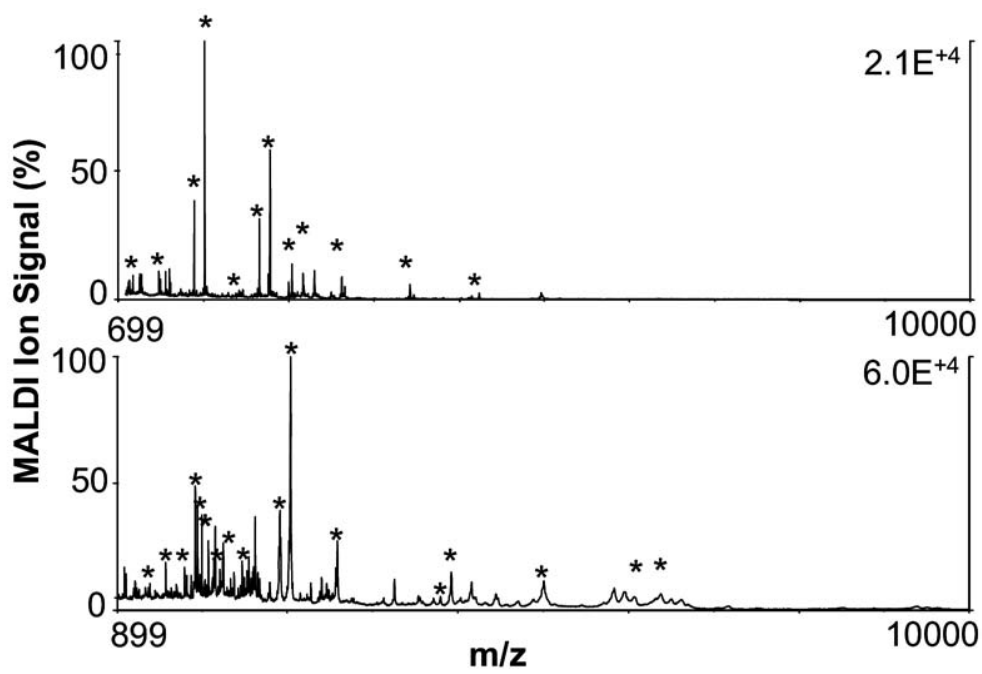

(b)

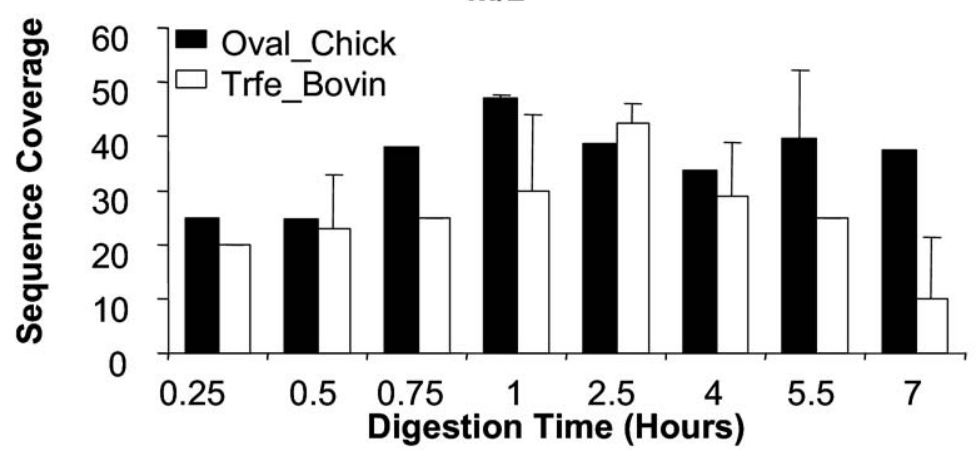

Figure 1. MALDI Mass spectra and detected sequence coverage for standard proteins digested in 10 $\mathrm{mg} / \mathrm{mL} \mathrm{CHCA}$. Spectra are shown for a half hour digestion of ovalbumin (a) and a $2.5 \mathrm{~h}$ digestion of transferrin (b). Asterisks mark identified peptide fragments matched to predicted $\mathrm{m} / \mathrm{z}$ values for peptides cleaved at aspartic acid residues. Sequence coverage from Mascot peptide mass fingerprint searches is plotted over time for both standard proteins; samples were analyzed in triplicate (c). 
shown here to focus solely on the utility of MALDI mass analysis. Mascot peptide mass fingerprint searches were used to compare the mass spectra from aliquots of these digests extracted at different times. As shown in Figure 1c for three replicate digestions, the sequence coverage for ovalbumin and transferrin are optimized between 1 and $2.5 \mathrm{~h}$ time points. For longer protein sequences, longer incubation times were more appropriate. Two-hour incubations worked better to achieve digestion of bovine transferrin.

\section{Comparison to Tryptic and Glu-C Digestions}

Comparison to enzymatic digestions was performed to additionally characterize the performance of acid hydrolysis. As an example, bovine transferrin was analyzed with the in silico proteomics experiment planner (IPEP) [20] to predict the efficiency of various digestions before experimental comparison. Mass spectrometry analysis of digests using trypsin, acid hydrolysis, and $S$. aureus V-8 endoprotease in bicarbonate buffer (specific cleavage to C-terminal to glutamic acid residues) can theoretically produce similar values for sequence coverage. Using IPEP, trypsin was found to have the highest number of peptides with 42 out of 83 peptides able to be detected using our MALDI settings $(\mathrm{m} / \mathrm{z} 750$ to 5500 ) resulting in $78.6 \%$ sequence coverage. Though acid hydrolysis produced fewer peptides, 33 out of 48 could be expected to be observed resulting in $85.5 \%$ sequence coverage. V-8 digestion produced 33 detectable peptides out of 45 total, providing $76.7 \%$ sequence coverage. These theoretical calculations show similar values for expected performance when using each type of proteolysis.

Both chicken egg ovalbumin and bovine transferrin were cleaved using CHCA, formic acid, trypsin, and $S$. aureus $\mathrm{V} 8$ protease in a total of six different methods (Figure 2). Acid hydrolysis was performed for $2 \mathrm{~h}$ using each of the following reagents: aqueous $2 \%$ formic acid, aqueous $2 \%$ formic acid with $50 \%$ acetonitrile, and 10 $\mathrm{mg} / \mathrm{mL}$ CHCA in aqueous 50\% acetonitrile. Trypsin digestion was studied at $2 \mathrm{~h}$ and overnight; V8 was examined in overnight digestions. Comparison of the sequence coverage obtained from each method for acid hydrolysis is shown in Figure $2 \mathrm{a}$ and $\mathrm{b}$ for transferrin and ovalbumin, respectively. At high amounts of protein, the sequence coverage achieved with CHCA hydrolysis was found to be comparable to digests using
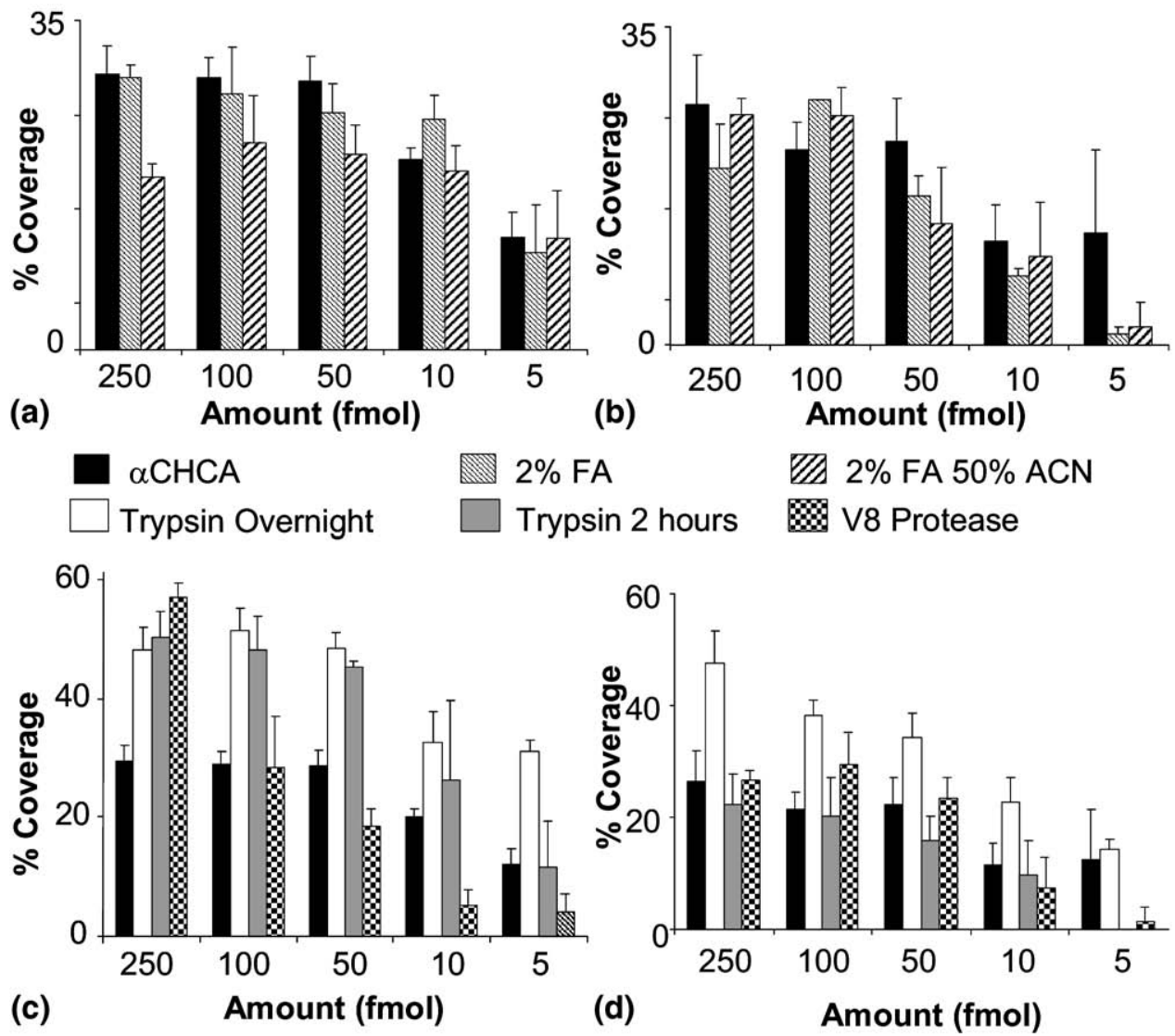

Figure 2. Comparison of sequence coverage obtained from acid hydrolysis and enzymatic digestions of bovine transferrin and chicken ovalbumin dilution series. Sequence coverage is plotted against amount of the digest deposited for all acid hydrolysis reactions of transferrin (a) and ovalbumin (b). CHCA digests are compared with enzymatic digests for the same proteins in (c) and (d). The key is relevant to all panels. 
Table 2. Detection of bovine ubiquitin and transferrin peptides following acid hydrolysis in CHCA. Proteins were digested in the presence of matrix and spotted on the MALDI target; the resulting Mascot score, sequence coverage, and number of peptides detected are shown

\begin{tabular}{|c|c|c|c|c|c|c|}
\hline \multirow[b]{2}{*}{$\begin{array}{c}\text { Amount } \\
\text { (fmol) }\end{array}$} & \multicolumn{3}{|c|}{ Ubiquitin } & \multicolumn{3}{|c|}{ Transferrin } \\
\hline & $\begin{array}{c}\text { Mascot } \\
\text { score }\end{array}$ & $\begin{array}{l}\text { Sequence } \\
\text { coverage }\end{array}$ & $\begin{array}{l}\text { Peptides } \\
\text { detected }\end{array}$ & $\begin{array}{c}\text { Mascot } \\
\text { score }\end{array}$ & $\begin{array}{l}\text { Sequence } \\
\text { coverage }\end{array}$ & $\begin{array}{l}\text { Peptides } \\
\text { detected }\end{array}$ \\
\hline 125 & 113 & $100 \%$ & 11 & 135 & $30 \%$ & 36 \\
\hline 25 & 90 & $100 \%$ & 9 & 134 & $29 \%$ & 31 \\
\hline 5 & 76 & $83 \%$ & 8 & 93 & $20 \%$ & 19 \\
\hline 2.5 & 64 & $70 \%$ & 7 & 23 & $12 \%$ & 8 \\
\hline 0.5 & 0 & $0 \%$ & 0 & 0 & $0 \%$ & 0 \\
\hline
\end{tabular}

either formulation with $2 \%$ formic acid. In ovalbumin digests, peptide cleaved by CHCA hydrolysis could be detected at lower amounts than those produced by formic acid as observed at $5 \mathrm{fmol}$ in Figure 2b. Ovalbumin peptides cleaved by matrix acid hydrolysis could be detected at $1 \mathrm{fmol}$ (data not shown). In comparison with the enzymatic digestions (trypsin and V-8 protease), acid hydrolysis of ovalbumin, and transferrin produced lower sequence coverage at higher concentrations (Figure $2 \mathrm{~b}$ and $\mathrm{d}$ ). However, using lower amounts of protein ( 5 to $10 \mathrm{fmol}$ ), the results from matrix digests were comparable to or better than the short tryptic digests and overnight V8 digests.

\section{Optimization of MALDI Matrix Digestion of Proteins}

The CHCA mediated proteolysis of ubiquitin was analyzed to study sensitivity, side reactions, and cleavage
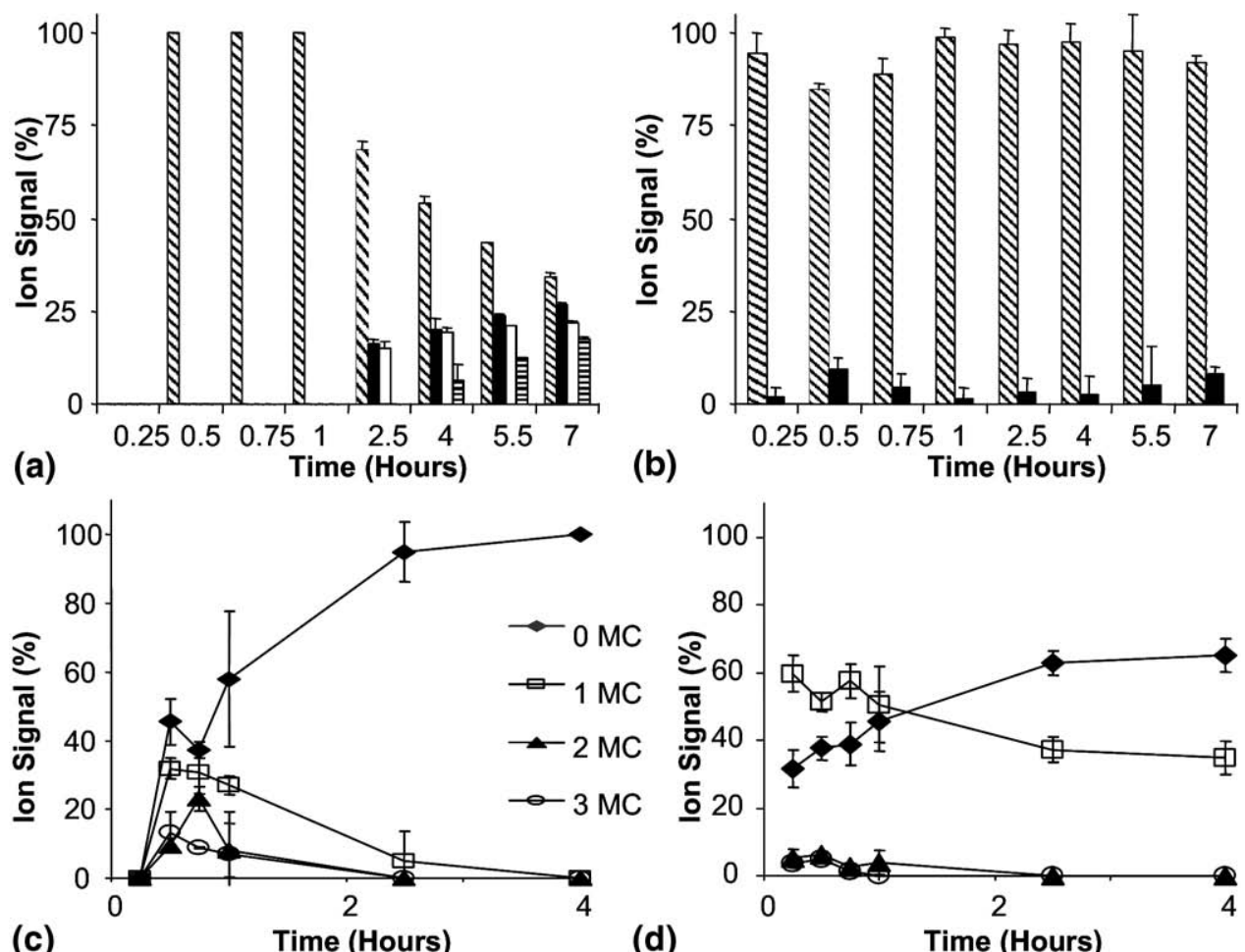

Time (Hours)

(d)

Time (Hours)

Figure 3. Ion signals detected for bovine ubiquitin hydrolyzed in $10 \mathrm{mg} / \mathrm{mL}$ CHCA. The peptides containing the N-terminus (a) showed complete conversion to the form with zero missed cleavages, MQIFVKTLTGKTITLEVEPSD. The peptides containing the C-terminus (b) were not cleaved as efficiently, but within the first hour, only the zero and one missed cleavage products, GRTLSDYNIQKESTLHLVLRLRGG and YNIQKESTLHLVLRLRGG, could be detected. The legend in the middle applies to both panels. Side reactions observed for two peptides show the relative amount of each ion signal is plotted for the intact peptide (diagonal stripes), loss of water (black), loss of aspartic acid (white), and both water and aspartic acid (horizontal stripes). (c) For the internal peptide, TIENVKAKIQDKEGIPPD, (c) increasing amounts of side reactions are detected. For the C-terminal peptide, YNIQKESTLHLVLRLRGG, (d) only a small amount of water loss is observed, which appears to be independent of reaction time. 
efficiency. Ubiquitin was selected because the intact molecule and the peptides produced by cleavage at the six aspartic acid residues can all be detected in a single linear mode MALDI mass spectrum. Furthermore, no disulfide bonds are present, so the protein should be completely denatured by the elevated temperature used for the acid hydrolysis. Ubiquitin proved to be an excellent substrate for acid hydrolysis with MALDI matrix. After 15 min incubations, several peptides [3-9] could be detected and manually assigned to the sequence. Complete sequence coverage from cleaved peptides could be attained at 45 to $60 \mathrm{~min}$ from as little as $5 \mathrm{fmol}$ of ubiquitin digest deposited on the plate. At $5 \mathrm{nM}$ ubiquitin concentrations, the sequence coverage began to drop. Five to seven peptides and only $70 \% \pm 21 \%$ of the sequence could be detected in three replicates with $5 \mathrm{nM}$ ubiquitin after $1 \mathrm{~h}$ of acid hydrolysis; the sequence coverage, Mascot scores, and the number of detected peptides are shown in Table 2. No signal for ubiquitin peptides could be detected in for $500 \mathrm{amol}$ deposited. To study proteolysis of a larger substrate protein, transferrin was hydrolyzed in CHCA solutions for $2.5 \mathrm{~h}$; MALDI peptide mass fingerprinting results are shown in Table 2. These digests yielded $\sim 30 \%$ coverage with the identification of 33 to 37 peptides and a Mascot Score of $135 \pm 12$ for $125 \mathrm{fmol}$ deposited on the MALDI target. As found with ubiquitin, all values decreased with the amount deposited with no detectable signal for 500 amol.

Similar to formic acid hydrolysis, specific side reactions became apparent at longer incubation times with
MALDI matrices: water loss and cleavage of the Cterminal aspartic acid from newly produced peptides. As shown in Figure $3 a$ and $b$, the ion signals corresponding to the internal ubiquitin peptide, TIENVKAKIQDKEGIPPD, illustrate the prevalence of these reactions. This cleaved peptide is not observed until after the $15 \mathrm{~min}$ time point. In reactions less than $2.5 \mathrm{~h}$, no side reaction products are detected. The only ion signal detected corresponds to the unmodified TIENVKAKIQDKEGIPPD peptide. Then, the water loss and cleavage of C-terminal aspartic acid increase over time. By $7 \mathrm{~h}$, the ion signal corresponding to the specific cleavage product makes up $\sim 30 \%$ of the total ion signal and the side reaction products are around $70 \%$. For the C-terminal peptide, YNIQKESTLHLVLRLRGG, water loss is observed at low amounts for the entire experiment, without significant change. This stability may be related to this specific sequence, but in general, the C-terminal sequences from proteins appear to undergo less degradation than $\mathrm{N}$-terminal or internal peptides.

While side reactions are minimized at short reaction times, the cleavage efficiency must also be evaluated. Using ubiquitin as the model system, the entire series of potential missed cleavages can be detected in the same spectrum. As shown in Figure $3 \mathrm{c}$ and $\mathrm{d}$, the production of N-terminal and C-terminal fragments can be comprehensively monitored. The relative amounts of each peptide are calculated using the peak height. After $1 \mathrm{~h}$ of incubation, the zero and one missed cleavage peptides at both the $\mathrm{N}$-terminus and $\mathrm{C}$-terminus are the
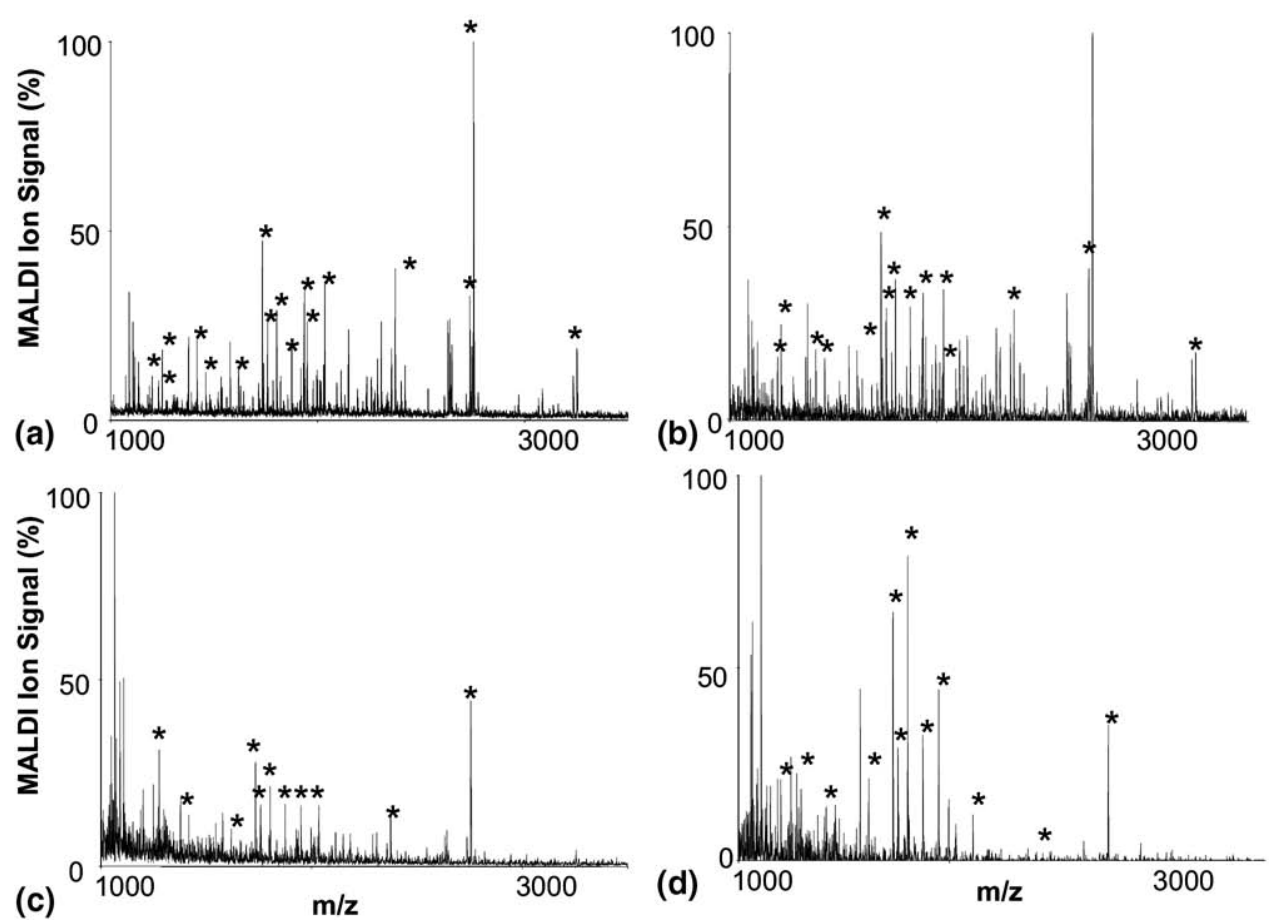

(c)

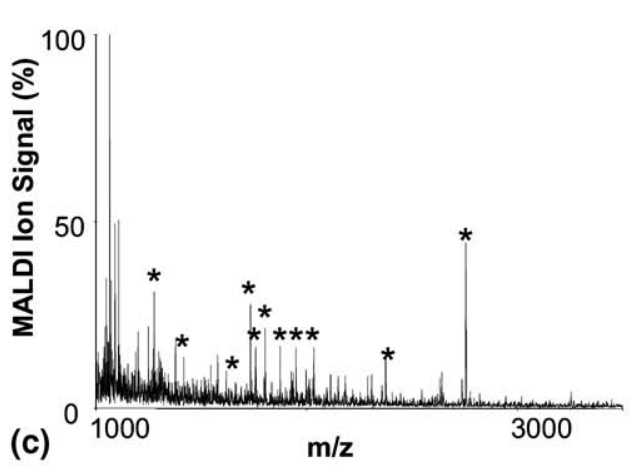

Figure 4. Minimal reaction volumes do not adversely affect MALDI mass spectra acquired after CHCA acid hydrolysis. Data are shown for $100 \mathrm{fmol} / \mu \mathrm{L}$ transferrin in (a) and (b) for full volume (100 $\mu \mathrm{L})$ and reduced volume $(5 \mu \mathrm{L})$ digests, respectively. Corresponding experiments were performed for reaction mixtures with $5 \mathrm{fmol} / \mu \mathrm{L}$ protein in (c) and (d). 
dominant species. The zero missed cleavage peptides, (N)-MQIFVKTLTGKTITLEVEPSD and YNIQKESTLHLVLRLRGG-(C), make up $\sim 50 \%$ of the total ion signal corresponding to peptides containing the $\mathrm{N}$ - and $\mathrm{C}$ terminus, respectively. By $4 \mathrm{~h}$, no other peptides containing the N-terminus are detected; the cleavage of the N-terminal sequence, MQIFVKTLTGKTITLEVEPSD, is complete. At 4h, YNIQKESTLHLVLRLRGG makes up $\sim 75 \%$ of the total ion signal for C-terminal peptides, and the sequence with one missed cleavage is the only other detectable peptide. Based on these observations and similar calculations for internal peptides, the $1 \mathrm{~h}$ timescales used for these reactions reduce side reactions, but enable sufficient cleavage efficiency.

\section{Minimization of Reaction Volumes}

Following the examination of digestion time, small reaction volumes were examined to minimize the amount of material necessary for detection. This step will be necessary to make acid hydrolysis in MALDI matrices competitive in analyzing biologic samples. Proteolysis of ubiquitin and transferrin were analyzed in $5 \mu \mathrm{L}$ digestion samples and compared with data acquired from $100 \mu \mathrm{L}$ reaction volumes. To prevent evaporation of the sample, low concentrations of glycerol $(1 \%)$ were added to the reaction mixture. For ubiquitin, the sequence coverage after $1 \mathrm{~h}$ of incubation was $100 \%$ in samples containing concentrations as low as $50 \mathrm{nM}$ protein in the reaction ( $25 \mathrm{fmol}$ deposited on the MALDI target). The lower concentrations, 10 and 5 $\mathrm{nM}$, yielded $86 \% \pm 24 \%$ and $53 \% \pm 20 \%$ sequence coverage, respectively. Even at these low concentrations, at least six specific peptides were consistently identified from ubiquitin. No ubiquitin peptides could be detected from reaction solutions containing $1 \mathrm{nM}$ ubiquitin (500 amol deposited). These values are comparable with those described above for $100 \mu \mathrm{L}$ reaction volumes with the same concentrations of ubiquitin. As shown in Figure 4, the number of peptide ion signals detected from high volume (left) and low volume (right) transferrin digests in $5 \mathrm{mg} / \mathrm{mL}$ CHCA were comparable at the $2 \mathrm{~h}$ time point. High concentration reaction mixtures (Figure $4 \mathrm{a}$ and $\mathrm{b}$ ) contained $1 \mu \mathrm{M}$ transferrin, and low concentrations (Figure 4c and d) had $5 \mathrm{nM}$ transferrin. The following signals were observed in both reactions at $1 \mu \mathrm{M}$ protein concentration: $m / z 1249.6,1417.7,1460.6,1617.9,1732.9,1757.8,1802.9$, $1820.9,1874.9,1935.9,1948.9,2034.0,2374.6,2735.5$, 2753.5, and 3332.7. Ten peptides were consistently de-
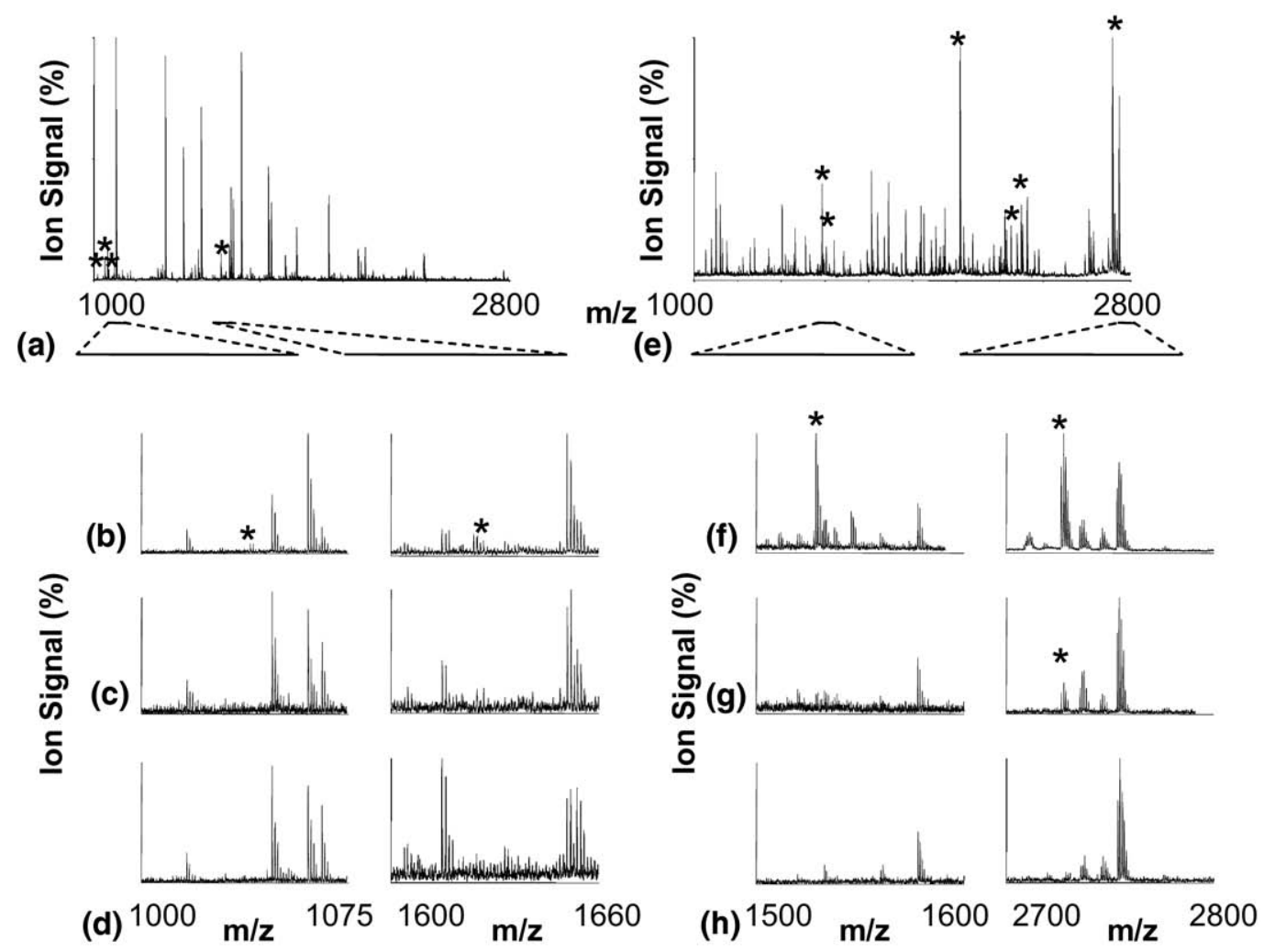

Figure 5. Detection of ubiquitin in low stoichiometry to transferrin in two h acid hydrolysis samples prepared in $5 \mathrm{mg} / \mathrm{mL}$ CHCA and overnight tryptic digestions. Samples containing $1 \mathrm{pmol} / \mu \mathrm{L}$ bovine transferrin were spiked with 100 (a), 50 (b), 10 (c), and zero (d) fmol/ $\mu \mathrm{L}$ bovine ubiquitin. Asterisks mark predicted peptide fragments that were identified for ubiquitin, which was detected even at levels as low as $1 \%$ the amount of transferrin in the mixture for both reactions. The insets show zoomed regions containing the ubiquitin peaks. 
tected in the $5 \mathrm{nM}$ protein reactions: $m / z$ 1249.6, 1417.7, 1617.9, 1732.9, 1757.8, 1820.9, 1874.9, 1948.9, 2034.0, and 2753.5. Although, the signal-to-noise ratios were slightly decreased, presumably because of the differences in the solvent system (i.e., the glycerol), the overall data quality was very similar for the two different reaction volumes.

\section{Detection of Minor Components in Mixtures}

To examine the detection of minor components in mixtures, ubiquitin was mixed with transferrin in low stoichiometries. This pair of proteins was chosen because ubiquitin hydrolysis produces few peptides, while transferrin digestion creates a complex background. Tryptic digestion (Figure 5a) is compared with matrix acid hydrolysis (Figure 5e) using ubiquitintransferrin mixtures. From tryptic digests, four peptides could be detected at $m / z$ 1039.5, 1067.6, 1081.6, and 1787.9 (marked with asterisks in Figure 5a). In acid hydrolysis of mixtures containing 10:1 ratios of transferrin to ubiquitin, specific peptides could be detected for ubiquitin at $m / z 1545.8,2097.2,2282.2,2350.3$, and 2726.5 (marked with asterisks in Figure 5e). Despite the lower concentration, the ion signal at $m / z$ 2097.2, which corresponds to YNIQKESTLHLVLRLRGG, is the base peak in the MALDI mass spectrum. Zoomed regions are shown for 10:1 mixtures (Figure 5b and f), 100:1 mixtures (Figure $5 \mathrm{c}$ and g), and transferrin controls for both digests (Figure $5 \mathrm{~d}$ and $\mathrm{h}$ ). In tryptic digests, the ubiquitin peaks cannot be detected when the ratio of transferrin to ubiquitin is increased. However, the signals at $\mathrm{m} / \mathrm{z} 2097.2$ and 2726.5, corresponding to C-terminal peptides with zero and one missed cleavage, persist even at 100:1 ratios of transferrin to ubiquitin with signal-to-noise ratios greater than 50 and 25, respectively. Successful detection of these peptides against the high transferrin background indicates that the method could be successful in identifying lower abundance components in mixtures. In the trypsin digestion duplicates, poorer sensitivity was achieved as shown by detection of the $m / z 1523.8$ peptide (corresponding to IQDKEGIPPDQQR) observed only down to a 50:1 transferrin to ubiquitin ratio. Other peptides, including $m / z 1039.5$ (EGIPPDQQR), were observed at lower transferrin to ubiquitin ratios $(10: 1)$.

\section{Application to In-Gel Hydrolysis of Immunoprecipitated $\beta$-Catenin}

In-gel matrix acid hydrolysis of standard proteins (400 fmol) and immunoprecipitated proteins resulted in identification though peptide mass fingerprinting and
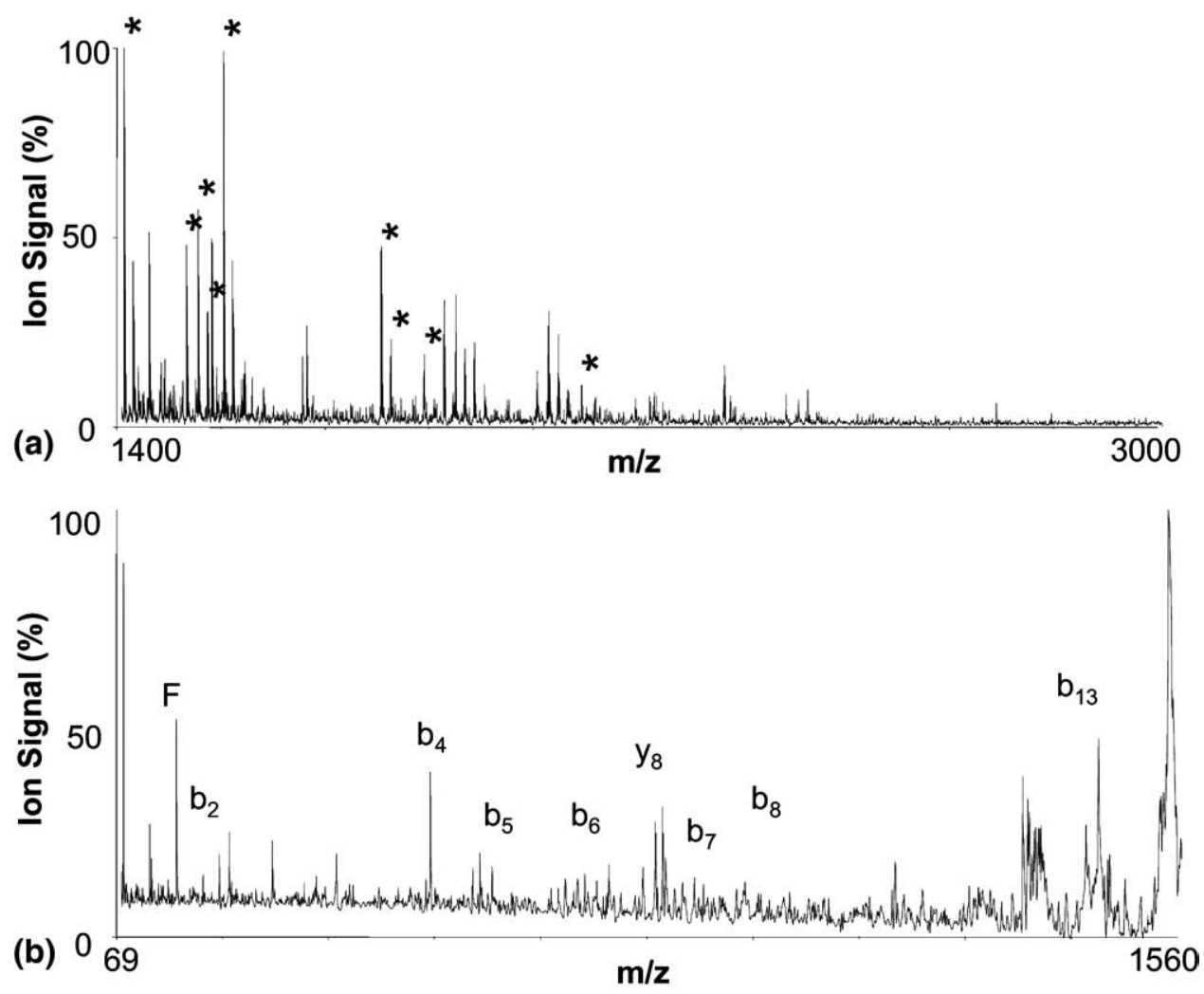

Figure 6. Peptides detected from CHCA hydrolysis of immunoprecipitated $\beta$-catenin. MALDI mass spectrum of peptides detected from CHCA hydrolysis of excised gel band (a). Asterisks mark identified peptides. The tandem mass spectrum (b) could be used to verify the sequence of the $\beta$-catenin peptide, PSYRSFHSGGYGQD, observed at $\mathrm{m} / \mathrm{z} 1557.7$. 
targeted tandem mass spectrometry. Protein identifications from samples hydrolyzed with CHCA were comparable to those obtained from $2 \%$ formic acid digestion. However, samples hydrolyzed in the matrix solution did not need to be diluted before MALDI analysis. Bovine ubiquitin could be identified with Mascot database searches with Score 127 based on 16 peptide assignments providing 100\% sequence coverage; bovine transferrin was assigned with a Score 104 with 28 peptides producing $31 \%$ sequence coverage. From the immunoprecipitated protein sample, positive identification of $\beta$-Catenin was confirmed by both peptide mass mapping and tandem MS/MS (Mascot Score $91,8 \%$ sequence coverage). The following peptide masses were specifically identified: $m / z$ 1403.7, 1500.7, 1518.7, 1539.4, 1557.7, 1816.7, 1868.0, and 2113.1 (Figure 6a). A tandem mass spectrum is shown for the PSYRSFHSGGYGQD peptide observed at $\mathrm{m} / \mathrm{z} 1557.7$ (Figure $6 b)$. This technique could be employed to verify the presence of specific protein targets and their binding partners.

\section{Conclusions}

Acid hydrolysis with MALDI matrices effectively cleaves peptides and proteins specifically and predictably at aspartic acid residues using a minimum of reagents and sample handling steps. At short timescales (less than $2 \mathrm{~h}$ ), these reactions produce minimal side reactions. However, they may not be appropriate for detection of post-translationally modified sequences, as shown by the dephosphorylation of synthetic phosphopeptides. Proteolysis in MALDI matrix is also compatible with analysis of low levels of analyte in small volumes and shows increased sensitivity for specific peptides in complex mixtures compared with tryptic digestion, as illustrated by the detection of basic peptides from ubiquitin against a background of transferrin peptides at higher concentrations. Positive identification of immunoprecipitated protein from in-gel acid hydrolysis shows a streamlined method for protein detection with less sample handling and the ability to retain a concentrated sample for MALDI analysis.

\section{Acknowledgments}

Proteomics at Moffitt is supported by the U.S. Army Medical Research and Materiel Command under Award no. DAMD17-022-0051 for a National Functional Genomics Center, the National
Cancer Institute under Award no. P30-CA076292 as a Cancer Center Support Grant, and the Moffitt Foundation. EGFR peptides were synthesized by Ted Gauthier at the Peptide and Mass Spectrometry Facility in the Chemistry Department of the University of South Florida. The authors thank Yi Chen for the $\beta$-Catenin immunoprecipitation.

\section{References}

1. Russell, W. K.; Park, Z.-Y.; Russell, D. H. Proteolysis in mixed organicaqueous solvent systems: Applications for peptide mass mapping using mass spectrometry. Anal. Chem. 2001, 73, 2682-2685.

2. Pramanik, B. N.; Mirza, U. A.; Ing, Y. H.; Liu, Y. H.; Bartner, P. L.; Weber, P. C.; Bose, A. K. Microwave-enhanced enzyme reaction for protein mapping by mass spectrometry: A new approach to protein digestion in minutes. Protein Sci. 2002, 11, 2676-2687.

3. Juan, H. F.; Chang, S. C.; Huang, H. C.; Chen, S. T. A new application of microwave technology to proteomics. Proteomics 2005, 5, 840-842.

4. Lin, S. S.; Wu, C. H.; Sun, M. C.; Sun, C. M.; Ho, Y. P. Microwaveassisted enzyme-catalyzed reactions in various solvent systems. J. Am. Soc. Mass Spectrom. 2005, 16, 581-588.

5. Lin, S.; Lin, Z.; Yao, G.; Deng, C.; Yang, P.; Zhang, X. Development of microwave-assisted protein digestion based on trypsin-immobilized magnetic microspheres for highly efficient proteolysis followed by matrix-assisted laser desorption/ionization time-of-flight mass spectrometry analysis. Rapid Commun. Mass Spectrom. 2007, 21, 3910-3918.

6. Fountoulakis, M.; Lahm, H.-W. Hydrolysis and amino acid composition of proteins. J. Chromatogr. A 1998, 826, 109-134.

7. Gobom, J.; Mirgorodskaya, E.; Nordhoff, E.; Hojrup, P.; Roepstorff, P. Use of vapor-phase acid hydrolysis for mass spectrometric peptide mapping and protein identification. Anal. Chem. 1999, 71, 919-927.

8. Li, A.; Sowder, R. C.; Henderson, L. E.; Moore, S. P.; Garfinkel, D. J.; Fisher, R. J. Chemical cleavage at aspartyl residues for protein identification. Anal. Chem. 2001, 73, 5395-5402.

9. Hua, L.; Low, T. Y.; Sze, S. K. Microwave-assisted specific chemical digestion for rapid protein identification. Proteomics 2006, 5, 586-591.

10. Zhong, H.; Marcus, S. L.; Li, L. Microwave-assisted acid hydrolysis of proteins combined with liquid chromatography MALDI MS/MS for protein identification. J. Am. Soc. Mass Spectrom. 2005, 16, 471-481.

11. Swatkoski, S.; Gutierrez, P.; Ginter, J.; Petrov, A.; Dinman, J.D.; Edwards, N.; Fenselau, C. Integration of residue-specific acid cleavage into proteomic workflows. J. Proteome Res. 2007, 6, 4525-4527.

12. Swatkoski, S.; Gutierrez, P.; Wynne, C.; Petrov, A.; Dinman, J.D.; Edwards, N.; Fenselau, C. Evaluation of microwave-accelerated residue-specific acid cleavage for proteomic applications. J. Proteome Res. 2008, 7, 579-586.

13. Swatkoski, S.; Russell, S. C.; Edwards, N.; Fenselau, C. Rapid chemical digestion of small acid-soluble spore proteins for analysis of Bacillus spores. Anal. Chem. 2006, 78, 181-188.

14. Swatkoski, S.; Russell, S.; Edwards, N.; Fenselau, C. Analysis of a model virus using residue-specific chemical cleavage and MALDI-TOF mass spectrometry. Anal. Chem. 2007, 79, 654-658.

15. Wang, N.; Mackenzie, L.; De Souza, A. G.; Zhong, H.; Goss, G.; Li, L. Proteome profile of cytosolic component of zebrafish liver generated by LC-ESI MS/MS combined with trypsin digestion and microwaveassisted acid hydrolysis. J. Proteome Res. 2007, 6, 263-272.

16. Reported by SciFinder 2007, calculated by Advanced Chemistry Development (ACD/Labs) Software V8.14 for Solaris 1994-2008.

17. Harris, D. C. Quantitative Chemical Analysis, 3rd ed.; W. H. Freeman and Co.: New York, 1991; pp. AP24-AP32.

18. Palmblad, M.; Cramer, R. Liquid matrix deposition on conductive hydrophobic surfaces for tuning and quantitation in UV-MALDI mass spectrometry. J. Am. Soc. Mass Spectrom. 2007, 18, 693-697.

19. Perkins, D. N.; Pappin, D. J.; Creasy, D. M.; Cottrell, J. S. Probabilitybased protein identification by searching sequence databases using mass spectrometry data. Electrophoresis 1999, 20, 3551-3567.

20. Lu, D.; Liu, R. Z.; Izumi, V.; Fenstermacher, D.; Haura, E. B.; Koomen, J.; Eschrich, S. A. IPEP: An in silico tool to examine proteolytic peptides for mass spectrometry. Bioinformatics 2008, 24, 2801-2802. 